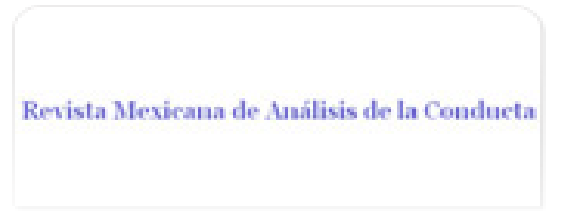

Revista Mexicana de Análisis de la Conducta ISSN: 0185-4534

editora@rmac-mx.org

Sociedad Mexicana de Análisis de la Conducta México

Menez, Marina; López, Florente

Variables Temporales como Determinantes de la Pausa Posreforzamiento

Revista Mexicana de Análisis de la Conducta, vol. 35, núm. 2, diciembre, 2009, pp. 57-73

Sociedad Mexicana de Análisis de la Conducta

Guadalajara, México

Disponible en: http://www.redalyc.org/articulo.oa?id=59311902005

- Cómo citar el artículo

- Número completo

- Más información del artículo

Página de la revista en redalyc.org

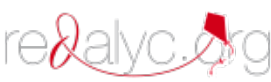

Sistema de Información Científica

Red de Revistas Científicas de América Latina, el Caribe, España y Portugal Proyecto académico sin fines de lucro, desarrollado bajo la iniciativa de acceso abierto 


\title{
Variables Temporales como Determinantes de la Pausa Posreforzamiento
}

\author{
Temporal Variables as Determinants \\ of the Post-Reinforcement Pause \\ MARINA MENEZ Y FLORENTE LÓPEZ ${ }^{1}$ \\ FACULTAD DE PSICOLOGÍA \\ UNIVERSIDAD NACIONAL AUTÓNOMA DE MÉXICO
}

\begin{abstract}
Resumen
En estado estable, los programas de razón e intervalo fijos generan un patrón de respuesta parecido: una pausa (PPR) proporcional al intervalo entre reforzadores (IER). Debido a esta relación, el IER fue considerado determinante de la pausa pero existe la posibilidad de que el tiempo de trabajo o la demora al reforzador también estén involucrados.

Estas variables generalmente están correlacionadas lo que dificulta evaluar su contribución relativa en la determinación de la pausa. Para solucionar parcialmente este problema, en este experimento se evaluó la contribución del IER y de la demora al reforzamiento como determinantes del tamaño de la pausa, utilizando programas de reforzamiento Encadenado RF1-TFx y Conjuntivo RF1-TFx, en un procedimiento acoplado. Los resultados indicaron que tanto el IER como la demora al reforzador fueron buenos predictores del valor de la PPR en estado estable y que la PPR en el programa Conjuntivo estuvo determinada temporalmente.
\end{abstract}

1. Esta investigación recibió financiamiento de los proyectos CONACYT 2008/89231, DGAPA IN305808 y por la beca PASPA-DGAPA otorgada al primer autor. Los autores contribuyeron en la misma proporción al diseño, realización y análisis de la investigación y agradecen los valiosos comentarios de tres revisores anónimos, que ayudaron a mejorar el escrito. La correspondencia puede enviarse a Av. Universidad 3004, Edif. D, Posgrado. Cubículo 24. Ciudad Universitaria, Col. Copilco-Universidad, CP. 04510, México, D. F., o a los correos: menez@servidor.unam.mx , florente@servidor.unam.mx

Recibido: Sept 15, 2009. Revisado: Octubre 20, 2009. Aceptado: Noviembre 11, 2009 
Palabras clave: intervalo entre reforzadores, demora al reforzador, programas acoplados, control temporal, ratas.

\begin{abstract}
Steady-state performance under both, Fixed-ratio and Fixed-interval reinforcement schedules generate a similar response pattern: a period with few or no responses, called postreinforcement pause (PRP), proportional to the interreinforcement interval (IRI). Because of this relationship, the IRI was considered as the determinant of the PRP, but the possibility exists that work time or delay to reinforcer are also involved. However, these variables are usually correlated, thus difficulting the evaluation of their relative contribution on the determination of the pause. To partially solve this problem, in the present experiment the contribution of the IRI and the reinforcer delay on the size of the pause was evaluated with a yoked procedure using Chained FR1-FTx and Conjunctive FR1-FTx reinforcement schedules. Results indicated that both IRI and delay to reinforcer were good predictors of the PRP value in steady state, and that the PRP in the Conjunctive schedule was temporally determined.
\end{abstract}

Keywords: interreinforcement interval, delay of reinforcement, yoked procedure, temporal control, rats.

Programas de reforzamiento como el Intervalo Fijo (IF), Tiempo Fijo (TF), Razón Fija (RF) y algunas de sus combinaciones, generan, en estado estable, un patrón de respuesta semejante que se caracteriza por un periodo con pocas o ninguna respuesta seguido por un periodo que finaliza con la entrega del reforzador, en el que las respuestas ocurren a una tasa relativamente constante o positivamente acelerada (Catania, 1970). La pausa posreforzamiento (PPR) o el tiempo desde el inicio del intervalo a la primera respuesta, proporciona un indicador de la transición entre estos dos estados. Por lo general se observa que el tamaño de la pausa es una proporción constante del valor del Intervalo entre Reforzadores (IER). Esto, junto con indicios de una independencia funcional entre los determinantes del valor de la pausa y de las tasas de respuesta global y terminal (Killeen, 1969; Dews, 1970; Shull, 1971; Rider y Kametani, 1984; Wynne y Staddon, 1988), llevó a algunos investigadores a atribuir la proporcionalidad PPR-IER a la regularidad temporal del IER, sobre todo en los programas IF.

Basándose en que el patrón de pausa-carrera ocurre tanto en programas IF como RF, Capehart, Eckerman, Guilkey y Shull (1980) intentaron determinar si el IER era la variable controladora. Contrastando programas de RF e IF, con IER comparables, estos autores reportaron pausas más pequeñas en 
programas de RF que en los de IF, para valores de IER menores a 30s (Experimento 1), aunque para un rango de 50 s a 100 s, las PPR fueron semejantes. Su análisis de las distribuciones de pausas por oportunidad permite saber si hay factores temporales implicados en la determinación de la pausa. En este análisis se determina el logaritmo de la probabilidad condicional de terminación de la pausa. Si la probabilidad es constante a lo largo del tiempo, se obtiene una función lineal decreciente; si la probabilidad de terminar la pausa es creciente, se obtiene una función cóncava hacia abajo pero si la probabilidad de terminar la pausa es decreciente, se obtiene una función convexa (con límite en pendiente 0). Los autores reportaron que, en el caso de los programas RF, estas funciones fueron principalmente lineales decrecientes mientras que en los programas IF fueron cóncavas hacia abajo. Este último resultado es consistente con una hipótesis de discriminación temporal i. e., la probabilidad de terminar la pausa incrementa con el tiempo transcurrido.

Sin embargo, en estado estable, la regularidad de la PPR en los programas IF hace que el tiempo de la primera respuesta a la entrega del reforzador sea relativamente constante. Por consiguiente, no se puede descartar la demora al reforzador como posible determinante del valor de la pausa (Shull, 1979). De acuerdo con este autor, el inicio de la carrera de respuesta (y por consiguiente, la PPR) en los programas IF y RF, está determinada por el tiempo entre la primera respuesta y la entrega del reforzador, o demora al reforzador. En estos casos, a medida que se incrementan los requisitos en uno y otro programa, la mayor demora determina una menor probabilidad de respuesta y, por consiguiente, un aumento del tiempo en que el animal empieza a responder. Lo anterior significaría que la PRP es un producto colateral del efecto de la demora del reforzador, no del IER (cf. Manabe, 1990).

Una tercera variable que podría afectar la duración de la pausa es el número de respuestas en el IER. Cuando se aumenta el valor de un programa de IF, también aumenta el número promedio de respuestas entre reforzadores (Wilson, 1954; Clark, 1958; Hanson, Campbell \& Witolawski, 1962; Dews, 1965 citados en Neuringer \& Schneider, 1968, aunque véase Wall, 1965 para un resultado contrario), al igual que lo hace la pausa. Por consiguiente, se establece una relación entre número de respuestas y tamaño de la PPR. Esto es más claro en los programas RF: a mayor el número de respuestas solicitado, mayor es la PPR. Aunque no debemos olvidar que los incrementos en requisito también generan cambios concomitantes en el IER, haciéndolo otro factor de interés (Ferster \& Skinner, 1957; Felton \& Lyon, 1966; Neuringer \& Schneider, 1968).

En resumen, la explicación de las regularidades observadas en la PPR en programas RF e IF, enfrenta el posible problema de confusión de variables. Como respuesta a esta dificultad, en varios estudios se intentó separar los efectos de IER y número de respuestas, pero los resultados no fueron concluyentes. Por un lado, Neuringer y Schneider (1968) compararon pro- 
gramas RF en los que mantenían constante el número de respuestas pero variaban el IER, con programas IF en los que mantenían constante el IER pero variaban el número de respuestas. Encontraron que únicamente los incrementos en el IER produjeron aumentos en la PPR, concluyendo que en ambos programas el IER es el determinante de la pausa. Por otro lado, otros investigadores encontraron que en programas de intervalo fijo, dependiendo de la duración del intervalo, el número de respuestas puede o no aumentar la pausa (Nunes, Alferink \& Crossman, 1979). En programas RF, los aumentos en la razón produjeron aumentos en la pausa pero en programas acoplados que igualaron los tiempos de trabajo o IER obtenidos y que mantuvieron el número de respuestas fijo, no se observaron cambios. De esta forma se confirmó el importante papel que juega el número de respuestas sobre la determinación de la PPR (Crossman, Heaps, Nunes \& Alferink, 1974).

Lo que se puede concluir es que en los programas de razón e intervalo fijos la pausa podría reflejar los efectos del IER, el tiempo de trabajo, la demora al reforzador o el número de respuestas emitidas durante este último período (Manabe, 1990). Es difícil determinar la importancia relativa de cada una de estas variables, porque como ya se describió, los cambios en una están correlacionados con cambios en las otras.

En un esfuerzo por evaluar la contribución relativa de las variables temporales IER y demora al reforzador en la determinación del valor de la pausa, en la presente investigación se comparó un programa Encadenado Razón Fija 1 Tiempo Fijo x (RF1 TFx) con un programa Conjuntivo Acoplado Razón Fija 1 Tiempo Fijo x (RF1 TFx), controlando por la variable número de respuestas (manteniendo el componente RF fijo en la unidad, en ambos programas). Esta preparación permitió comparar los efectos de manipular la demora al reforzador (componente TFx del programa encadenado) contra los efectos del IER (componente TFx del programa conjuntivo acoplado). La lógica es que al comparar estos programas se pueden aislar los efectos de demora (producidos por los incrementos en el requisito del TF en el programa encadenado) de los del IER (producidos por los incrementos en el IER en el programa conjuntivo).

\section{MÉTODO}

\section{Sujetos}

Seis ratas macho Wistar, provenientes del bioterio de la Facultad de Psicología (UNAM), con 3 meses de edad al inicio del experimento. Los animales fueron alojados en jaulas individuales, con acceso libre a agua. Se les privó de alimento a fin de mantenerlos al $80 \%$ de su peso bajo ingesta libre. Duran- 
te todo el experimento los animales estuvieron en una habitación con temperatura controlada, bajo un ciclo de $12 \mathrm{hrs}$. de luz y $12 \mathrm{hrs}$. de oscuridad. Las sesiones experimentales se corrieron durante el ciclo luminoso, a la misma hora del día, siete días a la semana.

\section{Aparatos}

Se utilizaron 4 cajas de condicionamiento operante con medidas $30.5 \mathrm{~cm}$ de ancho $\times 34 \mathrm{~cm}$ de profundidad $\times 35 \mathrm{~cm}$ de altura. Las paredes de la caja eran de aluminio. En la pared frontal se localizaron dos palancas fijas y tres luces indicadoras, colocadas sobre cada palanca y sobre el orificio de entrega del reforzador. Este orificio estaba ubicado al centro de la pared frontal, a $5 \mathrm{~cm}$ del piso de la caja. Por él se presentó una cucharilla elevadiza con una capacidad aproximada de $0.1 \mathrm{ml}$. En la parte superior de la caja, se localizó una bocina, que presentó un tono de aproximadamente $80 \mathrm{Db}$. Una computadora personal provista de una interfase MED controló los eventos dentro de la caja, registró las respuestas (tanto a la palanca derecha como a la izquierda) y los tiempos de ocurrencia de cada evento, con una aproximación a la décima de segundo.

El reforzador consistió en el acceso, durante $3 \mathrm{~s}$, a una solución de leche condensada diluida en agua, en una proporción de 1:2, que se presentó por medio de la cucharilla levadiza. Durante el reforzamiento, la cámara experimental se obscureció totalmente y se encendió la luz ubicada encima del orificio del dispensador de líquido.

\section{Procedimiento}

Los sujetos fueron moldeados manualmente a presionar la palanca izquierda. Se requirieron de dos a tres sesiones para que todos los sujetos adquirieran la respuesta de palanqueo. Cada sesión finalizó a la entrega del reforzador número 36.

Posteriormente se sometió a los sujetos a un programa de reforzamiento continuo durante dos sesiones. Enseguida todos los animales pasaron por las fases que se describen a continuación.

Fase 1. Asignación de parejas de sujetos. A fin de asegurar que las ejecuciones de los sujetos resulten comparables en términos de tasa de respuesta, se procedió a someter a todos los animales a un programa IV 15s, que permaneció vigente por dos sesiones. Después este valor se fue incrementando, en pasos de $15 \mathrm{~s}$ en 15s, hasta llevarlos al valor establecido de $60 \mathrm{~s}$, lo cual requirió de 8 sesiones en total. Después de permanecer por 15 sesiones bajo 
este último valor, se procedió a formar parejas de sujetos tomando como criterio que tuvieran una tasa de respuesta semejante.

Fase 2. Condiciones Experimentales. Una vez formadas las parejas, se asignó aleatoriamente a cada sujeto del par a una de dos condiciones: Condición Encadenado, consistente en un programa RF1 TFx, en el que el TF tomó los siguientes valores: $7.5,15,30,45$ o 60 segundos o Condición Acoplado, consistente en un programa Conjuntivo RF1 TFx, en el que el valor del TF estuvo determinado por el valor del Intervalo Entre Reforzadores (IER) producido por el sujeto asignado a la condición Encadenado (referidos en adelante como Encadenado y Conjuntivo Acoplado para facilitar las descripciones). Estas sesiones finalizaron a la entrega del reforzador número 36 o pasadas 2 horas y después de obtenido un reforzador, lo que ocurriese primero. Se determinó este criterio para mantener una duración de sesión razonable que permitiera correr a los seis sujetos en un tiempo máximo de seis horas, que incluyó el tiempo de la sesión y el tiempo requerido para el registro del peso de los animales, la prueba del equipo y su colocación y retiro de las cámaras operantes.

El orden de presentación de los valores TF para la condición Encadenado ( $\mathrm{y}$, consecuentemente, para los sujetos en la condición Conjuntivo Acoplado) se determinó aleatoriamente (véase Tabla 1).

Tabla 1

Orden de Presentación del TF, para las condiciones Encadenado (primer sujeto del par) y Conjuntivo (segundo sujeto del par). El número de sesiones en cada condición se presenta entre paréntesis

\begin{tabular}{cccc}
\hline & \multicolumn{3}{c}{ Valor del programa Tiempo Fijo } \\
\hline Valor del programa Razón Fija & Sujetos 2-5 & Sujetos 3-1 & Sujetos 4-6 \\
\hline 1 & $7.5(16)$ & $60(25)$ & $45(11)$ \\
1 & $45(25)$ & $7.5(17)$ & $30(25)$ \\
1 & $15(22)$ & $15(25)$ & $7.5(15)$ \\
1 & $30(25)$ & $45(22)$ & $60(11)$ \\
1 & $60(21)$ & $30(25)$ & $15(24)$ \\
\hline
\end{tabular}

La sesión se inició con el encendido de la luz general y la luz indicadora sobre la palanca izquierda. La primera respuesta en dicha palanca, activó un tono que señaló la demora al reforzador, de manera independiente para ambos sujetos de cada pareja. El objetivo de señalar la demora fue deprimir la ocurrencia de respuestas adicionales, pues como ya se mencionó, el procedimiento busca controlar el efecto del número de respuestas sobre la duración de la PPR. Las respuestas en la palanca derecha no tuvieron consecuencias 
programadas. Para los sujetos en la condición Encadenado, la demora al reforzador se mantuvo fija, mientras que para los sujetos en la condición Conjuntivo Acoplado, la demora varió dependiendo del momento en que emitían su primera respuesta. Los sujetos en esta condición obtuvieron el reforzador al mismo tiempo que el sujeto en la condición Encadenado, siempre y cuando, previo a este periodo, hubieran emitido al menos una respuesta, es decir, cuando cumplían el requisito del programa Conjuntivo en efecto (ver Figura 1). En caso de que los sujetos acoplados no emitieran respuesta alguna en este periodo, la luz general de la cámara se apagaba y permanecía así durante 3s (tiempo de reforzamiento para el animal en la condición Encadenado). El siguiente intervalo iniciaba inmediatamente después, siendo señalado por el restablecimiento de las condiciones de iluminación ya mencionadas.

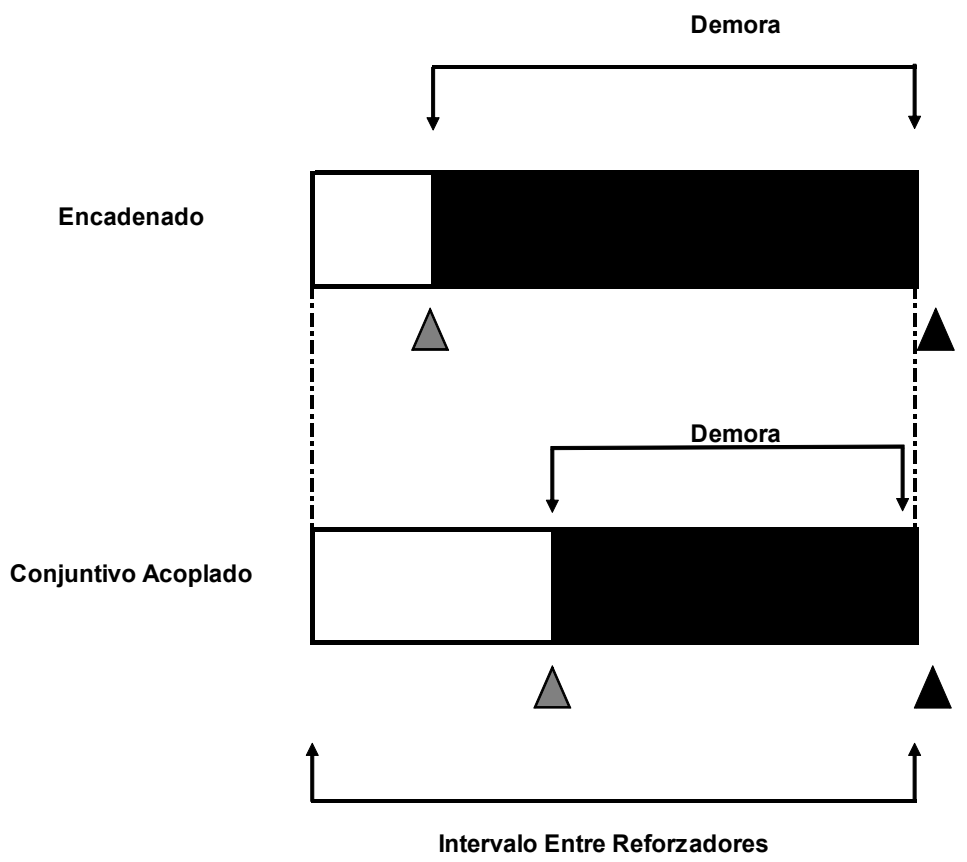

Figura 1. Diagrama del procedimiento acoplado. Los rectángulos en negro indican la demora al reforzador. Los rectángulos blancos la pausa. La suma de ambos, la duración del IER. El triángulo gris indica la respuesta y el triángulo negro el reforzador. 
El valor del TF se cambió cuando la ejecución de los sujetos en la condición Encadenado cumplió los siguientes criterios: un mínimo de diez sesiones y que el valor de la PPR promedio en las últimas cinco sesiones, se encontrará entre los valores mínimo y máximo de las mismas, o un máximo de 25 sesiones en el valor de TF.

\section{RESULTADOS}

Se realizó un análisis de la función que mejor describió la relación entre la PPR y el IER o la demora, para evaluar si (a) la relación PPR-IER es lineal creciente, (b) como suponen Capehart, et al, los valores de las pendientes asociadas a las dos condiciones difieren y (c) si la relación PPR-demora es lineal creciente. Para complementar lo anterior, se realizó un análisis de la distribución de pausas por oportunidad, que ayudaría a esclarecer si la PPR estuvo controlada por el tiempo.

Análisis de la relación PPR-IER. Dado que las sesiones terminaban por tiempo y además podía ocurrir que los sujetos de la condición Conjuntivo Acoplado no obtuvieran el reforzador programado, el número de observaciones varió entre condiciones y entre sujetos. Por consiguiente, para realizar el ajuste lineal se aplicó el siguiente criterio: cada condición debía contar con al menos el $55 \%$ de los ensayos reforzados ( 20 ensayos de 36 posibles, en los que los animales de cualquiera de las dos condiciones, obtienen el reforzador programado), en las últimas cinco sesiones. Esto dejó fuera la condición RF1 TF45s del sujeto 4 .

Un análisis exploratorio indicó un sesgo en la distribución de las PPR, por lo que el ajuste lineal se realizó sobre las medianas de la PPR y del IER.

Los ajustes lineales individuales (ver Tabla 2 ) resultaron estadísticamente significativos, excepto en un caso (sujeto 4, Encadenado, $p=.106$ ), y arrojan valores de $R^{2}$ de .83 a .99 , indicando que a medida que incrementó el IER mediano, también la PPR mediana aumentó. Las pendientes fueron muy variables y no todos los sujetos mostraron la tendencia predicha por Capehart et al: pendientes mayores en el programa Conjuntivo (ver Tabla 2, Figura 2). 
Tabla 2

Ajustes lineales a los valores medianos de la PPR en función del IER, para las condiciones Encadenado y Conjuntivo Acoplado. $N$ indica las condiciones, $R^{2}$ la varianza explicada y $p$ su probabilidad asociada

\begin{tabular}{llllcc}
\hline Suj & $N$ & Condición & Ajuste Lineal & $R^{2}$ & $p$ \\
\hline 2 & 5 & Encadenado & PPR $=-0.04+0.11^{*}$ IER & 0.97 & .002 \\
5 & 5 & Conjuntivo & PPR $=-5.63+0.40^{*}$ IER & 0.83 & .031 \\
3 & 5 & Encadenado & PPR $=-3.72+0.36^{*}$ IER & 0.91 & .012 \\
1 & 5 & Conjuntivo & PPR $=6.06+0.24^{*}$ IER & 0.94 & .005 \\
4 & 5 & Encadenado & PPR $=-3.16+0.29^{*}$ IER & 0.63 & .106 \\
6 & 4 & Conjuntivo & PPR $=2.86+0.41^{*}$ IER & 0.99 & .005 \\
\hline
\end{tabular}

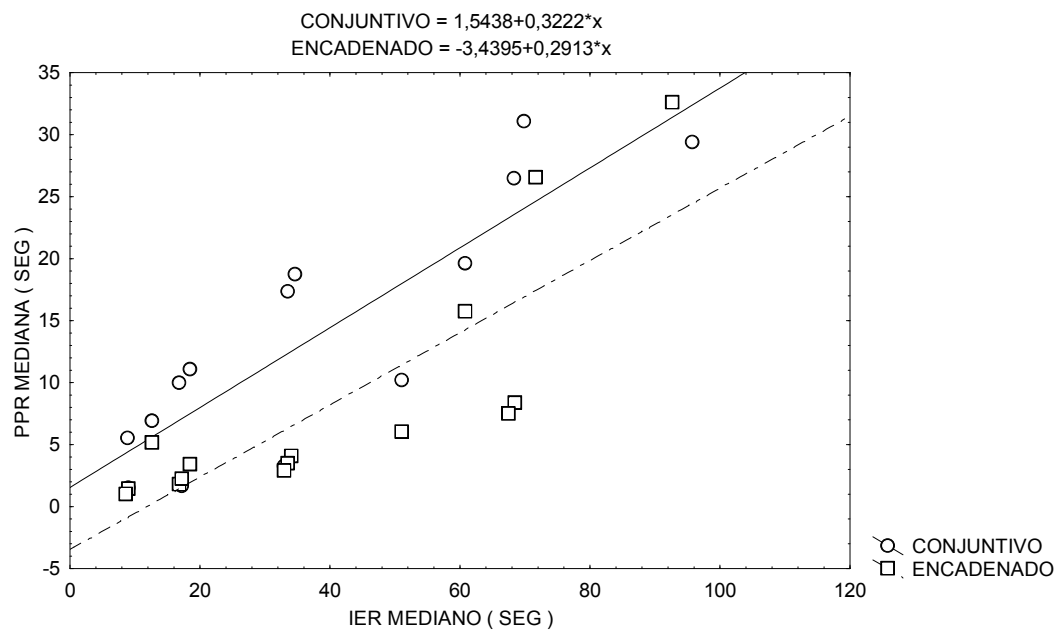

Figura 2. Ajuste lineal a los datos de PPR e IER medianos. Se analizan los datos de las cinco últimas sesiones en cada condición.

Se procedió a corroborar lo anterior con un análisis de regresión múltiple. La condición (Encadenado o Conjuntivo Acoplado) se codificó como variable dummy y se incluyó el termino de interacción IER*Condición, para evaluar si existieron diferencias entre las correspondientes ordenadas al origen. Los coeficientes asociados a estas variables no resultaron estadísticamente significativos (tCondición ${ }_{25,0.05}=-1.38, p=.178$; $\mathrm{tIER}^{*}$ Cond ${ }_{25,0.05}=-0.40$, 
$\mathrm{p}=.691$ ), indicando que las condiciones Encadenado y Conjuntivo Acoplado produjeron pausas de idéntico valor inicial y con idénticos incrementos para todos los valores de IER mediano analizados, con valor de pendiente .3. Se puede decir que la PPR mediana se inicia transcurrido aproximadamente un tercio del IER mediano, para valores comparables a los presentados en este estudio y que ambas funciones son bien descritas por la misma ecuación (PPR=15.54+0.32*IER).

Análisis de la relación PPR-demora. Para esta evaluación se utilizó la demora mediana como predictor de la PPR mediana, ajustándose una línea recta a los datos correspondientes a las cinco últimas sesiones de cada condición (valores constantes, en el caso de los sujetos en la condición Encadenado). Se aplicó el mismo criterio de inclusión de observaciones que en el análisis previo. Los ajustes individuales resultaron estadísticamente significativos excepto para los sujetos 5 (Conjuntivo Acoplado) y 4 (Encadenado). Como es de esperarse (dado que el IER incluye tanto a la demora como a la pausa) la cantidad de varianza explicada por la demora es inferior a la cantidad de varianza explicada por el IER ( $\mathrm{y}$ en un solo caso, correspondiendo al sujeto 2, igual; ver Tabla 3).

Tabla 3

Ajustes lineales a la PPR y demora (D) medianas, para las condiciones Encadenado y Conjuntivo Acoplado. N indica las condiciones, $R^{2}$ la varianza explicada y $p$ su probabilidad asociada

\begin{tabular}{llllcc}
\hline Suj & $N$ & Condición & Ajuste Lineal & $R^{2}$ & $p$ \\
\hline 2 & 5 & Encadenado & PPR $=-0.016+0.12^{*} \mathrm{D}$ & 0.97 & .003 \\
5 & 5 & Conjuntivo & PPR $=-5.712+0.51^{*} \mathrm{D}$ & 0.62 & .112 \\
\hline 3 & 5 & Encadenado & PPR $=-4.247+0.52^{*} \mathrm{D}$ & 0.78 & .045 \\
1 & 5 & Conjuntivo & PPR $=7.78+0.33^{*} \mathrm{D}$ & 0.91 & .011 \\
\hline 4 & 5 & Encadenado & PPR $=-0.56+0.28^{*} \mathrm{D}$ & 0.34 & .301 \\
6 & 4 & Conjuntivo & PPR $=5.02+0.61^{*} \mathrm{D}$ & 0.97 & .013 \\
\hline
\end{tabular}

El análisis grupal mostró que para ambos programas, la PPR mediana fue una función creciente de la Demora mediana y que sus pendientes no parecen ser distintas (Tabla 4). Esto se corroboró estadísticamente, pues la regresión múltiple con Condición como variable dummy y el término de interacción Condición ${ }^{*} D$, indicó que la misma ecuación describe la relación en ambos programas $\left(R^{2}=0.52, F_{(3,25)}=9.169, p<.001, b_{2}=b_{3}=0\right)$. 
Tabla 4

Ajustes lineales a la PPR y demora (D) medianas, para las

condiciones Encadenado y Conjuntivo Acoplado, considerando todos los

sujetos. $N$ indica el número de observaciones, $R^{2}$ la varianza explicada

y $p$ su probabilidad asociada

\begin{tabular}{lllcc}
\hline$N$ & Condición & Ajuste Lineal & $R^{2}$ & $p$ \\
\hline 15 & Encadenado & PPR $=-1.61+0.31 * \mathrm{D}$ & 0.42 & .009 \\
14 & Conjuntivo & PPR $=3.99+0.39 * \mathrm{D}$ & 0.54 & .003 \\
\hline
\end{tabular}

Adicionalmente, se efectuó un análisis de regresión para evaluar la posible contribución del número de respuestas. Se encontró que esta variable no resultó significativa de manera consistente, ni entre condiciones ni entre sujetos y que, en los casos en que alcanzó significancia, resultó ser parcialmente redundante con la variable IER.

En resumen, tanto el IER como la demora tienen una relación lineal creciente con la pausa, con una pendiente cercana a 0.3. Se debe mencionar que existió mucha variabilidad en los datos, por lo que este hallazgo debe interpretarse con precaución. Además, el número de respuestas no resultó ser una mejor predictora del valor de la PPR que el IER o la demora, por sí mismos.

Terminación de Pausas por Oportunidad. Se analizaron las distribuciones de las pausas por oportunidad (el logaritmo), pues en esta representación (a) una función lineal decreciente indica una probabilidad constante de terminación de la pausa a lo largo del tiempo, (b) una función cóncava hacia abajo indica una probabilidad creciente de terminar la pausa y (c) una función convexa indica una probabilidad decreciente de terminar la pausa. Las alternativas a y $b$ son las que se han asociado a las ejecuciones bajo los programas de razón e intervalo fijo, respectivamente y que en este estudio corresponden a los programas Encadenado y Conjuntivo Acoplado.

En las gráficas correspondientes a la condición Encadenado (Figura 3) se distinguen principalmente distribuciones con una probabilidad constante de terminación de la pausa a medida que transcurre el tiempo desde el reforzamiento (p.e., S-2 TF 7.5s, TF 45s, TF 60s; S-3 TF 45s, TF 60s; S-4 TF 45s). También se observan algunas distribuciones convexas (p.e., TF 30s, todos los sujetos). 

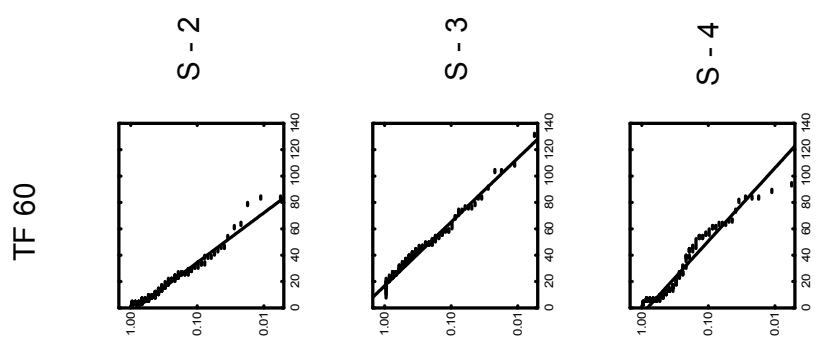

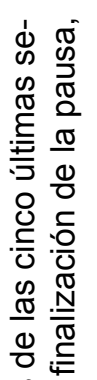
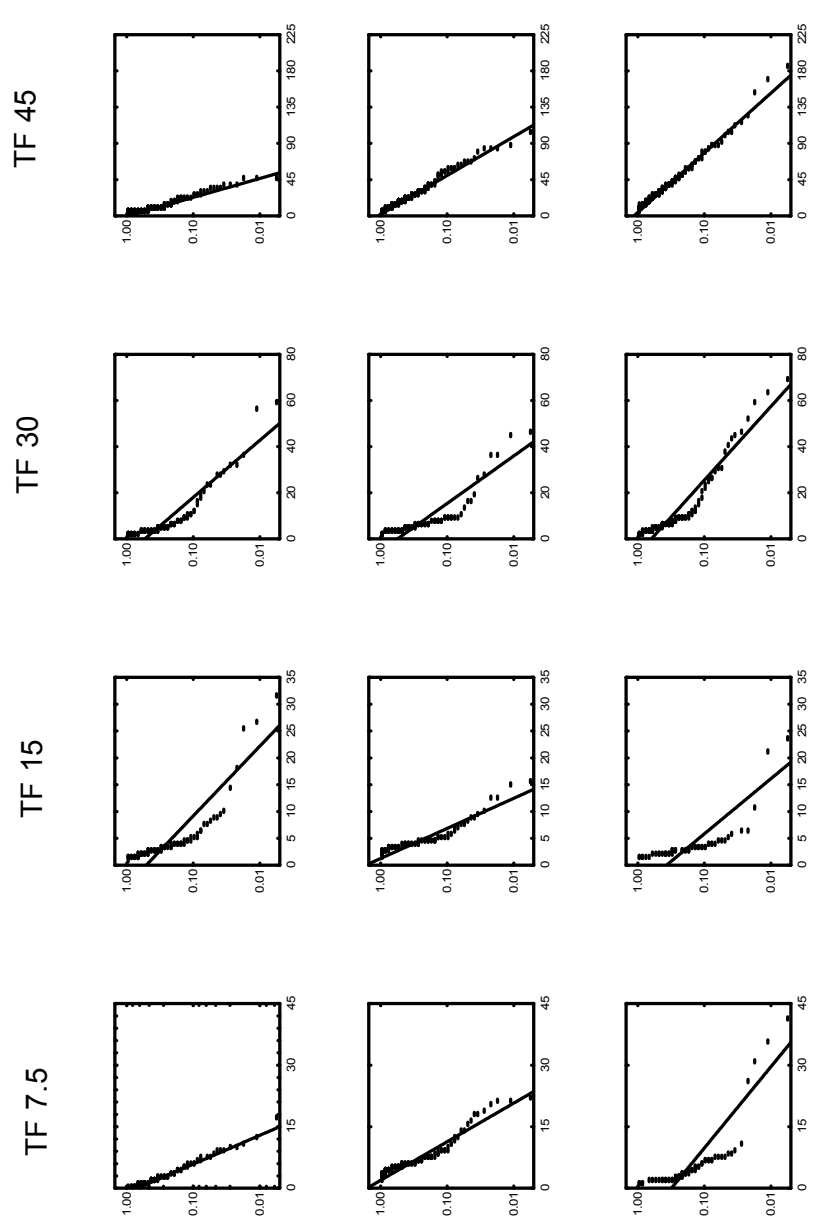

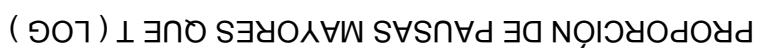

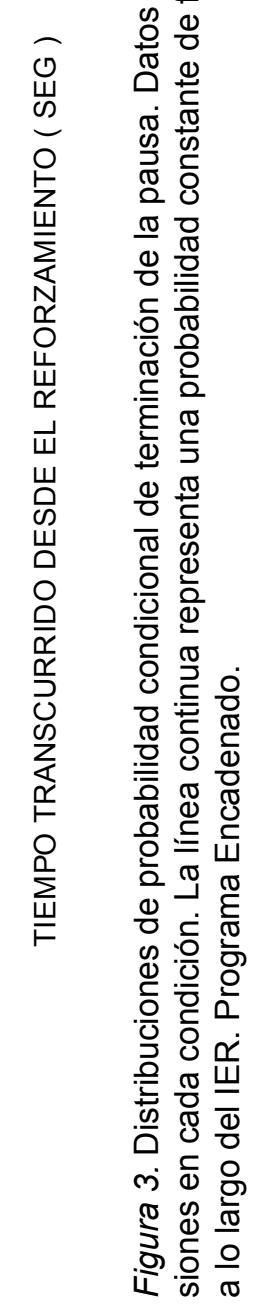



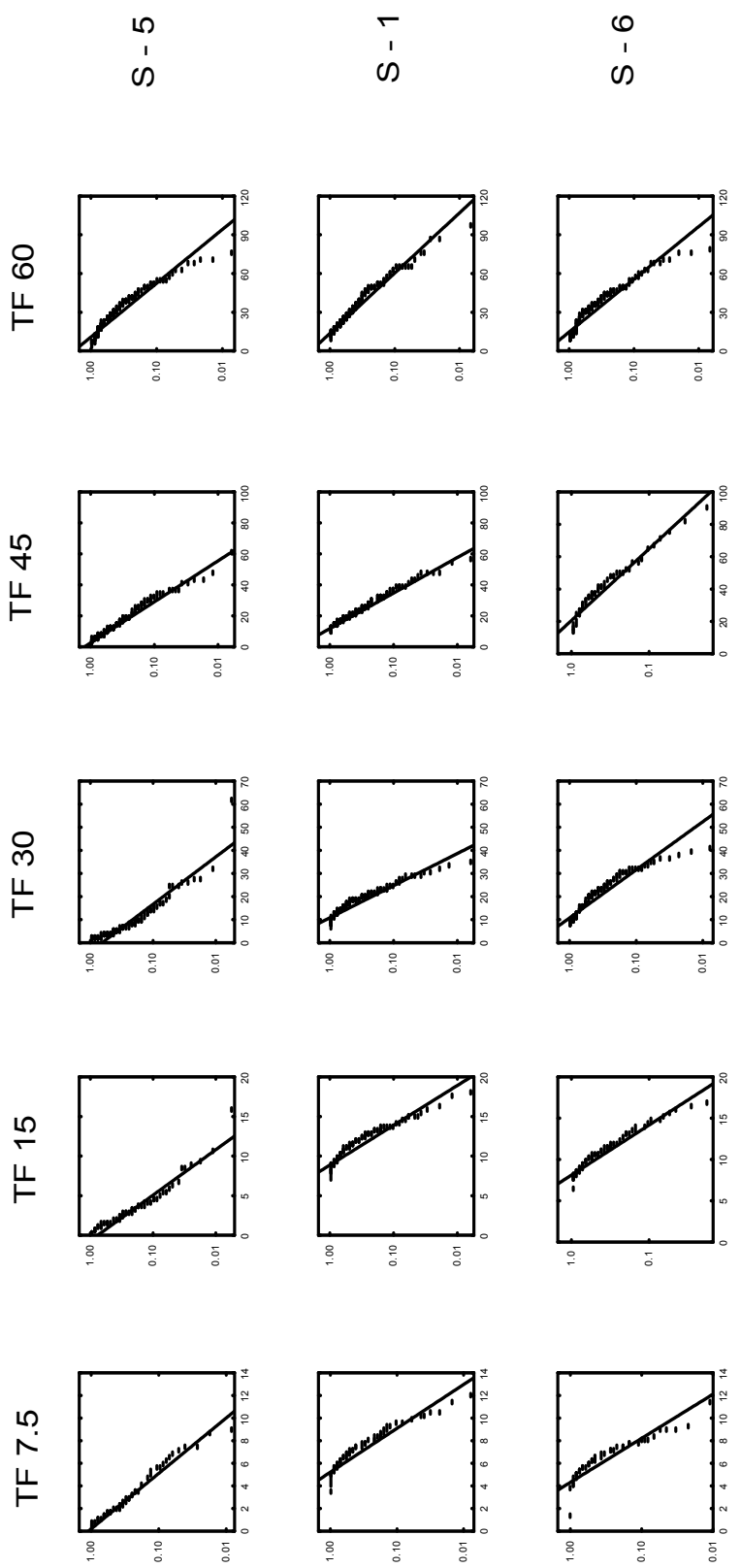

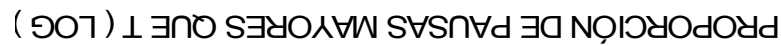

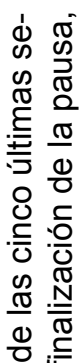

웡

苟

வ্

$\stackrel{\infty}{2}$

ฮ

즈 윰

응 음

:든 융

응

ह 을

$\stackrel{\Phi}{5}$

$\frac{0}{8} \stackrel{0}{\circ}$

त̄

ธิ㇒ 잉

은 흥 융

흥

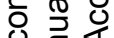

을

两

西 $\frac{5}{\circ}$

象

응

중

음

\& :응 항

응 잉

으 문

우

즘

फ

$\therefore \div$

중

홍 응으

i广 
En la condición Conjuntivo Acoplado se observan dos tipos de distribuciones: la que muestra una probabilidad creciente de terminar la pausa a medida que transcurre el tiempo desde el reforzamiento (p.e., S-5 TF 60s; S-1 TF 7.5s, TF 15s, TF 30s; S-6 todas las condiciones), y la que indica una probabilidad constante de terminar la pausa (p.e., S-5 TF $7.5 \mathrm{~s}$, TF 15s; S-1 TF 45s), con predominancia de las distribuciones del primer tipo.

\section{CONCLUSIONES Y DISCUSIÓN}

La relación lineal entre PPR e IER en programas con requisitos fijos, como el RF y el IF, fue considerada por algunos investigadores como resultado de la regularidad del intervalo entre reforzadores (Killeen, 1969; Dews, 1970; Shull, 1971; Rider \& Kametani, 1984; Wynne \& Staddon, 1988). Sin embargo, las contingencias en estos programas son diferentes: en los programas IF la entrega temporal del reforzador ocurre de manera relativamente constante, pero el número de respuestas por IER puede variar; en RF el número de respuestas es relativamente constante, pero el IER puede variar. Por consiguiente, resulta razonable considerar la posibilidad de que los determinantes de la pausa sean diferentes en estos programas. También es posible que un factor común a estos programas que contribuye a determinar el valor de la PPR no sea el IER. Por ejemplo, otra variable candidata a explicar esta relación es la demora al reforzador. Shull (1979) sugiere que las demoras al reforzador previamente experimentadas controlan la probabilidad momentánea del inicio del responder y, por consiguiente, controlan la PPR promedio. Esto ocurre porque si en el intervalo precedente hubo un tiempo de carrera corto, en el intervalo vigente se espera que la probabilidad momentánea incremente y viceversa. Esta dinámica establece condiciones distintas en los programas RF e IF: en los primeros los incrementos en pausas generan incrementos en el IER, mientras que en los segundos no lo hacen.

Son dos los problemas para decidir los factores comunes a la determinación de la PPR en los programas RF e IF: confusión de variables e interdependencia entre variables. Para resolver estos problemas, en el presente experimento se propuso un procedimiento que permitiera evaluar la contribución del IER y de la demora al reforzador en la duración de la PPR, manteniendo constante el requisito de respuesta. Esto se consiguió sometiendo a ratas a uno de dos programas: Encadenado (RF1 TFx) o Conjuntivo Acoplado (RF1 TFx), donde el componente TF del encadenado correspondió a la demora al reforzador. Los resultados indicaron que tanto el IER como la demora al reforzador fueron predictores del valor de la pausa, la cual abarcó el primer tercio del intervalo entre reforzadores. 
Los análisis efectuados para evaluar la contribución independiente del IER y de la demora, según los ajustes lineales, no fueron concluyentes: tanto la demora como el IER fueron buenos predictores de la duración de la pausa. Por consiguiente, los resultados no permiten afirmar que uno $u$ otro fue el determinante principal de la misma. Sí se encontró una relación lineal entre el tamaño de la PPR y el valor del IER. Sin embargo, Capehart et al (1980) y Manabe (1990) reportan que las pausas generalmente son más cortas en los procedimientos en que el reforzador se hace depender de la demora que en los que se hace depender del IER. Los resultados de este estudio no son congruentes con lo obtenido por estos autores: las pendientes obtenidas para las condiciones encadenado y conjuntivo acoplado no difirieron entre sí. Sin embargo, los resultados sí fueron congruentes con los hallazgos de Wynne y Staddon (1988). En conclusión, considerando los hallazgos anteriores, los resultados del presente estudio parecen indicar una relación más compleja entre los programas que entregan reforzadores a tiempos relativamente constantes y la PPR. En el presente caso, por ejemplo, debe considerarse que la PPR ocurrió en el primer tercio del intervalo, contra la observación más común que reporta entre un medio y dos tercios. Debe ser claro, no obstante, que la demora señalada del presente estudio determinó prácticamente una demora real i. e. la distancia temporal efectiva entre la respuesta y la ocurrencia del reforzador, debido a las pocas respuestas emitidas durante el estímulo. Adicionalmente debe considerarse que la regularidad temporal de la entrega del reforzador en programas IF, no se cumple en los programas encadenados, que solo establecen un mínimo. Por estas razones y otras señaladas por Keenan (1999), cabe esperar una relación dinámica entre los factores constantes y variables involucrados en las contingencias relativamente periódicas.

El análisis de la probabilidad condicional de terminación de la pausa (distribución de pausas por oportunidad) corroboró un hallazgo reportado por Capehart, et al, (1980): en el programa Conjuntivo Acoplado (semejante al IF) la probabilidad de terminación de la pausa decreció a medida que transcurrió el tiempo entre reforzadores, mientras que en el programa Encadenado, (similar al RF), esta probabilidad se mantuvo constante. Lo anterior significa que las manipulaciones del presente experimento fueron exitosas en el sentido de reproducir las contingencias temporales que caracterizan a los programas de razón e intervalo fijos. Si consideramos únicamente la relación entre ejecución y los factores temporales de la programación, llegamos a la conclusión de que el programa Conjuntivo Acoplado propició el desarrollo de discriminación temporal. Esto se puede ver con claridad en que, en este programa, las curvas de probabilidad condicional de terminación de la pausa fueron cóncavas hacia abajo, mientras que en el Encadenado no, aunque sí produjo algunas curvas semejantes a las reportadas bajo programas de 
RF. Este resultado es consistente con el análisis de las contingencias que establecen uno y otro programa y que descartan la posibilidad de un control semejante del IER sobre la PPR en ellos (Capehart, et al, 1980; Manabe, 1990)

Los enfoques modernos sobre la ejecución en programas temporales subraya el papel que juega el tiempo transcurrido desde un marcador, que puede ser el reforzador mismo como en los programas IF (Guilhardi \& Church, 2005). De hecho, se ha considerado al tiempo como la variable fundamental de la conducta (Zeiler, 1998) puesto que es la dimensión en que se despliegan todos los eventos. La presente investigación ilustra uno de los frentes en que se considera esta dimensión: cuáles son las distribuciones y las relaciones temporales entre eventos psicológicamente importantes y cómo contribuyen de manera dinámica al control de la conducta.

\section{REFERENCIAS}

Capehart, G. W. Eckerman, D. A. Guilkey, M, \& Shull, R. L. (1980). A comparison of ratio and interval reinforcement schedules with comparable interreinforcement times. Journal of the Experimental Analysis of Behavior, 34, 61-76.

Catania, A. C. (1970). Reinforcement schedules and psychophysical judgments: A study of some temporal properties of behavior: En W. N. Schoenfeld (Ed.). The theory of reinforcement schedules, (pp. 1-42). New York: Appleton-Century-Crofts.

Crossman, E. K., Heaps, R. S., Nunes, D. L., \& Alferink, L. A. (1974). The effects of number of responses on pause length with temporal variables controlled. Journal of the Experimental Analysis of Behavior, 22, 115-120.

Dews, P. B. (1970). The theory of fixed-interval responding. En W. N. Schoenfeld (Ed.), The theory of reinforcement schedules (pp. 43-61). New York: Appleton-Century-Crofts.

Felton, M., \& Lyon, D. O. (1966). The postreinforcement pause. Journal of the Experimental. Analysis of Behavior, 9, 131-134.

Ferster, C. B., \& Skinner, B. F. (1957). Schedules of Reinforcement. Appleton-CenturyCrofts.

Guilhardi, P., \& Church, R. M. (2005). Dynamics of temporal discrimination. Learning and Behavior, 33 (4), 399-416.

Keenan, M. (1999). Periodic response-reinforcer continguity: temporal control but not as we know it. Psychological Record, 49, 273-297.

Killeen, P. (1969). Reinforcement frequency and contingency as factors in fixed-ratio behavior. Journal of the Experimental Analysis of Behavior, 12, 391-395.

Manabe, K. (1990). Determinants of pigeons' waiting time: effects of interreinforcement interval and food delay. Journal of the Experimental Analysis of Behavior, $53,123-132$. 
Neuringer, A. J., \& Schneider, B. A. (1968). Separating the effects of interreinforcement time and number of interreinforcement responses. Journal of the Experimental Analysis of Behavior, 11, 661-667.

Nunes, D. L., Alferink, L. A., \& Crossman, E. K. (1979). The effects of number of responses on the postreinforcement pause in fixed-interval schedules. Journal of the Experimental Analysis of Behavior, 31, 253-257.

Rider, D. P., \& Kametani, N. H. (1984). Interreinforcement time, work time, and the postreinforcement pause. Journal of the Experimental Analysis of Behavior, 42, 305-319.

Shull, R. L. (1971). Postreinforcement pause duration on fixed-interval and fixed-time schedules of food reinforcement. Psychonomic Science, 23 (1B), 77-78.

Shull, R. L. (1979). The postreinforcement pause: some implications for the correlational law of effect. En M. D. Zeiler y P. Harzem (Eds.). Advances in Analysis of Behavior, Vol. 1, Reinforcement and the organization of Behavior (pp. 193-221) Chichester: John Wiley and Sons.

Wall, A. M. (1965). Discrete-trials analysis of fixed-interval discrimination. Journal of Comparative and Physiological Psychology, 60 (1), 70-75.

Wynne, C. D. L., \& Staddon, J. E. R. (1988). Typical delay determines waiting time on periodic-food schedules: static and dynamic tests. Journal of the Experimental Analysis of Behavior, 50, 197-210.

Zeiler, M. D. (1998). On sundials, springs, and atoms. Behavioural Processes, 44, 89-99. 